\title{
REVISITANDO A AQUISIÇÃO DO ATAQUE RAMI- FICADO CCV: ESTÁGIOS DO DESENVOLVIMENTO SILÁBICO
}

Andressa Toni

(USP/CNPq)

\section{RESUMO}

Este artigo trata de discutir a manifestação de estágios de desenvolvimento no percurso de aquisição de sílabas de ataque ramificado CCV (Consoante + +Consoante + +Vogal) do Português Brasileiro - estrutura presente em palavras como 'bruxa', 'blusa', 'prato', 'placa'. Retomando os resultados de Teixeira (1988), Lamprecht (1993), Ávila (2000), Ribas (2002), Staudt (2008) e Baesso (2009), o artigo examina possíveis motivações à observação (ou não) de estágios intermediários no desenvolvimento infantil. Investiga-se se as motivações podem residir i) numa tendência geral do percurso de aquisição fonológica - tal como observado na aquisição de outras estruturas silábicas, como a coda $\mathrm{CVC}$; ii) no desenvolvimento individual de cada criança; ou iii) em efeitos metodológicos relativos ao tipo de coleta de dados. Para verificar tais motivações, analisam-se dados naturalísticos de 3 sujeitos, acompanhados quinzenalmente, e dados experimentais de 49 sujeitos, obtidos num teste de repetição de palavras e pseudopalavras. Os resultados apontam que o tipo de coleta de dados pode afetar a observação de estágios do desenvolvimento silábico em contexto naturalístico, uma vez que o desbalanceamento da quantidade de alvos CCV e de sua combinação fonotática pode afetar a determinação de estratégias como a seleção lexical e os alvos/direção das substituições em CCV. O desenvolvimento individual mostrou-se correlacionado às tendências fonológicas gerais assinaladas por Teixeira (1988) e Lamprecht (1993), à medida que tais generalizações podem atuar na limitação dos diferentes percursos possíveis à criança na aquisição $\mathrm{CCV}$. O estudo sugere, ainda, que o tipo de estratégia empregado em reparo ao CCV também depende do desenvolvimento silábico infantil.

PALAVRAS-CHAVE: Fonologia; Aquisição de linguagem; Estágios de desenvolvimento; Sílaba; Ataque ramificado 


\section{Introdução: 0 que são estágios?}

A presença de estágios de desenvolvimento configura-se como um paradigma usual no percurso de aquisição fonológica: elementos linguísticos como o modelo prosódico inicial das palavras, o inventário segmental ou os tipos silábicos não se descortinam à criança de forma integral, tal como um conjunto maciço, mas de maneira gradual, fracionada, organizada e, muitas vezes, dependente daquilo que foi adquirido nos estágios anteriores. Observa-se, por exemplo, que os tipos silábicos (como CV, V, CVC, CCV) são adquiridos em etapas, segundo uma determinada ordem, que pode variar a depender da língua e da frequência dessas estruturas silábicas. Observa-se, ainda, que mesmo as diferentes combinações fonotáticas de um dado tipo silábico também não se revelam de maneira conjunta na fala infantil, sendo igualmente adquiridas de forma gradual e dependente do padrão de frequência da sua língua, conforme ilustrado em (1), relativamente à aquisição da coda silábica e das classes consonantais licenciadas nesta posição (MATZENAUER \& MIRANDA, 2012, p. 110):

(1) Língua

Holandês

Catalão e Espanhol

Português Europeu

Português do Brasil

Autor
Fikkert (1994)
Borrás (2008)
Freitas (1997)
Matzenauer-Hernandorena (1990)

Autor

Fikkert (1994)

Freitas (1997)

Matzenauer-Hernandorena (1990)

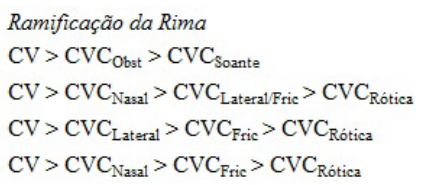

Ramificação da Rima

$\mathrm{CV}>\mathrm{CVC}_{\text {Obst }}>\mathrm{CVC}_{\text {Soante }}$

$\mathrm{CV}>\mathrm{CVC}_{\text {Nasal }}>\mathrm{CVC}_{\text {Lateral/Fric }}>\mathrm{CVC}_{\text {Rótica }}$

$\mathrm{CV}>\mathrm{CVC}_{\mathrm{Nasal}}>\mathrm{CVC}_{\mathrm{Fric}}>\mathrm{CVC}_{\mathrm{Rótica}}$

Exemplo 1

Como observado em (1), as sílabas de tipo CVC não emergem na fala da criança já contendo todas as combinações fonotáticas permitidas em sua língua. Este descompasso entre estrutura e segmentos pode ser tomado como um argumento à Teoria da Sílaba de Selkirk (1982), que sugere que a sílaba, enquanto conceito linguístico, pode ser decomposta em dois elementos: i) o molde silábico, que corresponde à sua estrutura, formada por constituintes organizados hierarquicamente nos nós binários [Ataque, Rima[Núcleo, Coda]], e que são, por sua vez, ramificáveis; e ii) os filtros segmentais, que representam os segmentos que podem ser associados a cada nó terminal do molde silábico. Ambos os elementos, molde e filtros, devem ser adquiridos pela criança em seu desenvolvimento fonológico.

Segundo Tessier (2006), os estágios presentes no percurso de aquisição da linguagem caracterizam-se como períodos intermediários entre o início e o fim do desenvolvimento linguístico infantil, em que se tem a captura parcial da totalidade de propriedades do elemento-alvo da crian- 
ça. Embora tais períodos intermediários sejam, em geral, regulares - isto é, semelhantes na fala de diversas crianças, tanto aquelas inseridas numa mesma língua como também em comparação ao percurso de desenvolvimento de línguas distintas, como observado em (1) -, estes estágios de aquisição são também permeados pela variação individual dos próprios sujeitos, como salienta Lamprecht (2004) - variação que pode dar-se "tanto em termos de idade de aquisição como também quanto aos caminhos percorridos - as estratégias de reparo ${ }^{1}$ utilizadas - para atingir a produção adequada" (LAMPRECHT, 2004, p. 25).

\begin{tabular}{|c|c|c|c|c|c|c|}
\hline & \multicolumn{3}{|c|}{ Posiçãio tônica } & \multicolumn{3}{|c|}{ Posição átona } \\
\hline & Alvo & Produção infantil & Glosa & Alvo & Produçào infantil & Glosa \\
\hline $\begin{array}{l}\text { Esićgio intetal } \\
\text { Ramificaçoes reduzidas } \\
(1 ; 0.28-1 ; 09.01)\end{array}$ & $\begin{array}{l}\text { /kxa.'kxa/ } \\
\text { /plces/ } \\
\text { /flces/ }\end{array}$ & $\begin{array}{l}\text { [ka.'kx] } \\
\text { [poe:] } \\
\text { [ßße:] }\end{array}$ & $\begin{array}{l}\text { 'Cracra' } \\
\text { 'chorar' } \\
\text { 'flor' }\end{array}$ & $\begin{array}{l}\text { /bsi'ze/ } \\
\text { /absi'ko/ }\end{array}$ & $\begin{array}{l}\text { [boc'çi:] } \\
\text { [pupse'ko] }\end{array}$ & $\begin{array}{l}\text { 'quebrado' } \\
\text { 'damasco' }\end{array}$ \\
\hline $\begin{array}{l}\text { Estógio intermediàrio } \\
\text { Ramificaçoes em } \\
\text { posiçầo tônica } \\
(1 ; 09.29-2 ; 03.05) \\
\end{array}$ & $\begin{array}{l}\text { /bi.'bs } \\
\text { fglis/' } \\
\text { /si.'txuj/ } \\
\text { /ploes/ }\end{array}$ & 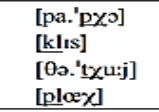 & $\begin{array}{l}\text { 'garrafa' } \\
\text { 'deslizar' } \\
\text { 'abóbora' } \\
\text { 'chorar' }\end{array}$ & $\begin{array}{l}\text { ffusi.'go/ } \\
\text { fbsy.'le/ } \\
\text { fgli.'sad' } \\
\text { ftxu.'ve/ }\end{array}$ & $\begin{array}{l}\text { [bu.'ko] } \\
\text { [br.'le] } \\
\text { [ka.'saed] } \\
\text { [tu.'ve] }\end{array}$ & $\begin{array}{l}\text { 'geladeira" } \\
\text { 'queimado' } \\
\text { 'deslizar' } \\
\text { 'achar' } \\
\end{array}$ \\
\hline $\begin{array}{l}\text { Esiógio finat } \\
\text { Ramificaçoes produzidas } \\
(2 ; 03.15)\end{array}$ & /'grol & ['gво] & 'grande' & $\begin{array}{l}\text { /txu.'ve/ } \\
\text { 'plaje/ }\end{array}$ & $\begin{array}{l}\text { [txu.'ve] } \\
\text { ['pläfe] }\end{array}$ & $\begin{array}{l}\text { 'achar' } \\
\text { 'chào' }\end{array}$ \\
\hline
\end{tabular}

Aplicados ao nível da sílaba, os estágios intermediários definem-se como o período entre o momento inicial da aquisição de um tipo silábico, representado pela sua ausência no sistema fonológico infantil, e o momento final de seu percurso de desenvolvimento, que consiste na produção da sílaba tal como na língua alvo. Estes estágios intermediários caracterizam-se pelo uso sistemático de determinadas adaptações input-output, ou estratégias de reparo, que podem ser aplicadas visando alterar tanto o molde silábico - p.ex., a estrutura CCV - quanto visando modificar o contexto fonotático da sílaba almejada pela criança - como a combinação entre as oclusivas e as líquidas $/ 1, r /$.

Para ilustrar a presença de estágios intermediários, Tessier (2006) toma como exemplo a aquisição do ataque ramificado em Francês (Quadro 1), em que se observa um estágio em que somente sílabas CCV em posição tônica são produzidas como o alvo pela criança, havendo apagamento da consoante $\mathrm{em}_{2}$ nas posições átonas: 


\begin{tabular}{|c|c|c|c|c|c|c|}
\hline & \multicolumn{3}{|c|}{ Posição tônica } & \multicolumn{3}{|c|}{ Posição átona } \\
\hline & Alvo & Produção infantil & Glosa & Alvo & Produção infantil & Glosa \\
\hline $\begin{array}{l}\text { Estágio inicial } \\
\text { Ramificações reduzidas } \\
(1 ; 00.28-1 ; 09.01)\end{array}$ & 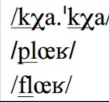 & 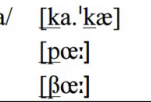 & $\begin{array}{l}\text { 'Chacra' } \\
\text { 'chorar' } \\
\text { 'flor' }\end{array}$ & $\begin{array}{l}\text { / } \underline{\mathrm{b}_{\mathrm{B}}} \mathrm{i}^{1} \mathrm{ze} / \\
\text { /absi'ko/ }\end{array}$ & $\begin{array}{l}{\left[\underline{b} \gtrless^{\prime} c ̧ i:\right]} \\
{[\text { pupææko] }}\end{array}$ & $\begin{array}{l}\text { 'quebrado' } \\
\text { 'damasco' }\end{array}$ \\
\hline $\begin{array}{l}\text { Estágio intermediário } \\
\text { Ramificações somente } \\
\text { em posição tônica } \\
(1 ; 09.29-2 ; 03.05)\end{array}$ & 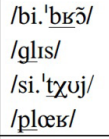 & 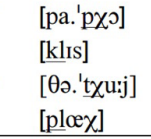 & $\begin{array}{l}\text { 'garrafa' } \\
\text { 'deslizar' } \\
\text { 'abóbora' } \\
\text { 'chorar' }\end{array}$ & $\begin{array}{l}\text { /fвi.'go/ } \\
\text { /bsy.'le/ } \\
\text { /gli.'sad/ } \\
\text { /txu.'ve/ }\end{array}$ & $\begin{array}{l}\text { [bu.'ko] } \\
\text { [b.'le] } \\
\text { [ka.'sæd] } \\
\text { [tu.'ve] }\end{array}$ & $\begin{array}{l}\text { 'geladeira' } \\
\text { 'queimado' } \\
\text { 'deslizar' } \\
\text { 'achar' }\end{array}$ \\
\hline $\begin{array}{l}\text { Estágio final } \\
\text { Ramificações realizadas } \\
(2 ; 03.15)\end{array}$ & /'gБо/ & ['gво] & 'grande' & $\begin{array}{l}\text { /t文u.'ve/ } \\
\text { /'plã } \int \mathrm{e} /\end{array}$ & 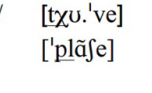 & $\begin{array}{l}\text { 'achar' } \\
\text { 'flor' }\end{array}$ \\
\hline
\end{tabular}

Quadro 1: Estágios na aquisição do ataque ramificado em Francês (TESSIER, 2006, p. 409, adaptado)

Relativamente ao percurso de aquisição do ataque ramificado em Português Brasileiro, contudo, diferentes resultados são relatados na literatura acerca da presença de tais estágios intermediários. Tendo isso em vista, o presente artigo propõe-se retomar as observações e divergências referidas nessa literatura, assim como contribuir com tais estudos trazendo novos dados, tanto de coleta naturalística como de metodologia experimental, a fim de cogitar e discutir possíveis fatores de influência aos resultados apresentados. O objetivo do artigo consiste, assim, em analisar a observação destes estágios de desenvolvimento ao longo da aquisição da sílaba CCV.

$\mathrm{O}$ trabalho está organizado como se segue: na seção 2 adiante, a literatura relativa à aquisição do ataque ramificado em PB será apresentada, e as divergências nela contidas acerca da presença ou ausência de estágios no desenvolvimento das sílabas CCV serão discutidas. Com base nessa exposição, serão aventadas possíveis causas a estas divergências, que serão analisadas conforme os dados descritos pela seção seguinte, 3 . Em 4 tem-se a discussão dos resultados da pesquisa, que serão contrastados àqueles referidos na literatura. Em 5 , por fim, nossas considerações finais acerca do percurso de desenvolvimento do ataque ramificado em Português Brasileiro.

\section{Aquisição do ataque ramificado em Português Brasileiro: Literatura}

De acordo com Lamprecht et al (2004), as estruturas silábicas permitidas no sistema fonológico do PB têm sua aquisição consolidada por volta dos 5 anos, seguindo uma ordem de estabilização como a ilustrada na Figura 1 abaixo (adaptada de RIBAS, 2002): 


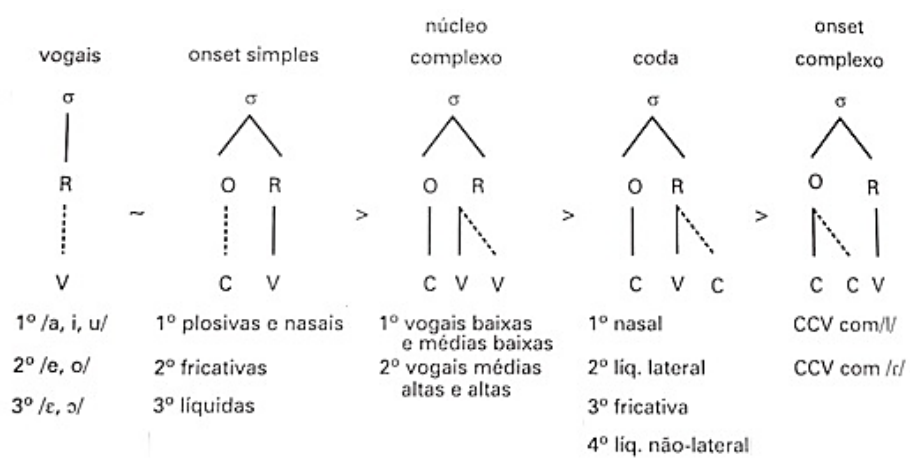

Figura 1: Estágios do desenvolvimento silábico segundo Ribas (2002)

Embora a sílaba CCV seja uma estrutura de aquisição tardia, o surgimento das primeiras produções-alvo do ataque ramificado é registrado já em fases iniciais da fala infantil, aos 1;9 anos no corpus de Ribas (2002), e 1;7 anos em Toni (2016). Neste período entre o surgimento e a aquisição $\mathrm{CCV}$, é consenso na literatura que a redução do ataque ramificado (por meio do apagamento de $\mathrm{C}_{2}$ : $\mathrm{CCV}>\mathrm{C}_{1} \mathrm{~V}$, como em ['bu.fe] 'bruxa', ['bu.ze] 'blusa') seria a forma de adequação input-output mais utilizada pela criança na tentativa de produzir o CCV.

Além da redução $\mathrm{CCV}>\mathrm{C}_{1} \mathrm{~V}$, também são mencionadas na literatura outras estratégias de reparo não tão recorrentes na fala da criança: a epêntese, a metátese, a transposição, a coalescência, as substituições segmentais em $\mathrm{C}_{1}$ ou $\mathrm{C}_{2}$ e o apagamento (cf. Quadro 7 na seção 3.3). Estas estratégias, quando sistemáticas, podem constituir estágios no desenvolvimento silábico, uma vez que podem indicar um reconhecimento da ramificação de ataque que seria anterior ao domínio da produção $\mathrm{CCV}$, propriamente. Por exemplo, uma criança que, na tentativa de produzir um CCV como /br/, produz formas como ['bur.je] 'bruxa' (metátese) ou [ba'ra.vv] 'bravo' (epêntese), embora demonstre ainda não ter domínio sobre a produção do molde $\mathrm{CCV}$, manifesta um reconhecimento de que esta sílaba é composta por dois segmentos que devem ser produzidos em sequência. Da mesma forma, ao produzir formas como ['bla.so] 'braço' ou ['bru.ze] 'blusa', a criança demonstra reconhecer a estrutura CCV e a propriedade [+soante] dos elementos em $\mathrm{C}_{2}$, mas ainda não ter domínio fonético e/ou fonológico sobre as líquidas que ocupam esta posição silábica.

Tratando de estágios intermediários, dois tópicos devem ser observados à discussão: o tipo de segmento a constituir o ataque ramificado 
- que poderia configurar um estágio em que apenas uma das líquidas é produzida como o alvo pela criança, por exemplo, ocorrendo aplicação sistemática de estratégias de substituição $\mathrm{C}_{2}$ - e o tipo de estrutura empregado antes da produção-alvo do $\mathrm{CCV}$ - em que haveria estratégias visando alterar CCV a CVC, como na metátese; CCV a CV.CV, como na epêntese; ou visando alterar a posição acentual do CCV, como na transposição, por exemplo.

Em relação a um possível padrão à produção das líquidas $/ 1, r /$ no ataque ramificado, notam-se discordâncias na literatura, embora a metodologia de elicitação dos dados em geral tenha sido semelhante em diversos estudos. Para Ávila (2000) e Baesso (2009), analisando dados elicitados com o instrumento fonoaudiológico Avaliação Fonológica da Criança, a líquida lateral emergiria antes da líquida não-lateral em posição CCV no sistema fonológico infantil, de forma análoga à ordem da aquisição segmental. Por outro lado, para Teixeira (1988), observando dados transversais de 11 crianças, embora a líquida lateral se produza em $\mathrm{CCV}$ antes da líquida não-lateral, este momento seria precedido por um estágio em que ocorreria substituição de ambas as líquidas pelo glide /j/, bem como sucedido, também, por um estágio em que o padrão inverso da produção das líquidas ocorreria, havendo hipergeneralização de C/1/V a C[r]V. Lamprecht (1993) e Galea (2008) encontram em seus dados padrão semelhante a Teixeira (1988): a substituição em $\mathrm{C}_{2}$ tanto pode ocorrer em direção $\mathrm{C} / \mathrm{r} / \mathrm{V}>\mathrm{C} / \mathrm{l} / \mathrm{V}$ quanto em direção contrária, $\mathrm{C} / \mathrm{l} / \mathrm{V}>\mathrm{C} / \mathrm{r} / \mathrm{V}$. Já para Ribas (2002), num estudo transversal que também utilizou o instrumento Avaliação Fonológica da Criança, com o total de 80 participantes, e Staudt (2008), num estudo longitudinal acompanhando 8 crianças, ambas as líquidas emergiram na estrutura ramificada em tempos semelhantes; desta forma, a aquisição do ataque complexo seguiria um percurso diferente das demais estruturas silábicas, que licenciam seus segmentos um a um no nó terminal na sílaba. Este percurso de licenciamento simultâneo seria motivado, para as autoras, pelo término do processo de aquisição segmental no momento de estabilização dos ataques ramificados - diferentemente do momento de estabilização dos demais tipos silábicos adquiridos pela criança, anteriores à $\mathrm{CCV}$.

Também se observam divergências na literatura relativamente aos estágios intermediários estruturais na aquisição do CCV - tanto em estudos relativos ao Português Brasileiro como na comparação entre Português Brasileiro, Português Europeu e Holandês. Trazendo inicialmente 
estudos sobre o PB, destacamos que, segundo Teixeira (1988), no percurso de aquisição CCV também podem ocorrer, paralelamente às substituições $\mathrm{C}_{2}$, as estratégias de epêntese, metátese e, numa última etapa pré-aquisição, transposições sistemáticas a fim de produzir o CCV no início da palavra, como em [dre'gaw] 'degrau'. A autora pontua, contudo, que estes estágios não seriam obrigatórios na aquisição fonológica, mas sim tendências gerais no percurso aquisitivo - ou seja, podem ser sistemáticos na fala de uma criança, mas não regularmente observáveis numa comparação entre sujeitos. Por outro lado, Ribas (2002) e Staudt (2008) afirmam não haver estágios intermediários na aquisição do ataque ramificado, havendo passagem direta da produção reduzida $\mathrm{C}_{1} \mathrm{~V}$ à sua produção correta $\mathrm{CCV}$, uma vez que, em seus dados, menos de $10 \%$ das palavras sofreram reparos diferentes da redução $\mathrm{CCV}$, o que indicaria a ausência de um padrão de substituições sistemáticas da estrutura CCV a tipos CVC ou CV.CV por exemplo.

Salientamos, no entanto, que Ribas (2002) se aproxima a Teixeira (1988) ao apontar ser possível reconhecer quatro tendências ou fases distintas no percurso de aquisição $\mathrm{CCV}$, em que seriam observadas características peculiares a cada momento do desenvolvimento do ataque ramificado - embora não sistemáticas nem regulares -, como descrito pelo Quadro 2 a seguir.

\begin{tabular}{|c|c|c|c|}
\hline Fase & Faixa etária & $\begin{array}{c}\text { \% de produções- } \\
\text { alvo CCV }\end{array}$ & Descrição \\
\hline 0 & $1 ; 0$ a $1 ; 11$ & $\begin{array}{l}1 /: 0 \% \\
/ \mathrm{s} /: 1 \%\end{array}$ & $\begin{array}{l}\text { Reparos voltados à } C_{1} \text { : coalescência, substituição } C_{1} \text {, apagamento } \\
\mathrm{CCV} \text { ou } \mathrm{C}_{1} \mathrm{C}_{2} \text {, além da redução } \mathrm{CCV}>\mathrm{C}_{1} \mathrm{~V}\end{array}$ \\
\hline 1 & $2 ; 0$ a $3 ; 1$ & $\begin{array}{l}1 / \text { de } 0 \% \text { a } 33 \% \\
/ \mathrm{r} /: \text { de } 0 \% \text { a } 15 \%\end{array}$ & $\begin{array}{l}\text { Reparos visando ambiente facilitador, em direção à } C_{1} \text { labial ou } \\
\text { tônica na ausência da labial: substituição das liquidas e das } \\
\text { obstruintes, metátese, movimento reciproco }\end{array}$ \\
\hline 2 & $3 ; 2$ a $4 ; 0$ & $\begin{array}{l}1 / \text { de } 0 \% \text { a } 69 \% \\
/ \mathrm{s} /: \text { de } 22 \% \text { a } 71 \%\end{array}$ & $\begin{array}{l}\text { Reparos visam evitar } \mathrm{C}_{1} \text { coronal metáteses e movimentos reciprocos } \\
\text { buscam } \mathrm{C}_{1} \text { labial ou tônica na ausência da labial }\end{array}$ \\
\hline 3 & $4 ; 2$ a $5 ; 3$ & $\begin{array}{l}1 / \text { de } 29 \text { a } 100 \% \\
/ \mathrm{s} /: \text { de } 39 \% \text { a } 95 \%\end{array}$ & Poucas estratégias de reparo: epêntese, metátese \\
\hline
\end{tabular}

Quadro 2: Fases na aquisição CCV segundo Ribas (2002, adaptado)

Observa-se no Quadro 2 que na Fase 0 apenas o elemento em posição $C_{1}$ é alvo de estratégias de reparo, sugerindo uma possível ausência do segmento em posição $\mathrm{C}_{2}$ na representação das palavras produzidas pela criança. Exemplos como [mĩ'ka] 'brincar' e [mĩ'ke.to] 'brinquedo' 
(Ribas, 2002, p. 90) favorecem este argumento uma vez que o espraiamento de nasalidade deveria ser barrado pela presença de uma consoante entre /b/ e /i/. Por sua vez, nas Fases de 1 a 3 tem-se o emprego de estratégias estruturais e segmentais de modificação da sílaba CCV, semelhantes àquelas apontadas por Teixeira (1988).

É interessante destacar, ainda, que poucos são os estágios ou tendências referentes ao desenvolvimento estrutural do ataque ramificado sugeridos pela literatura acima apresentada, que parece verificar em seus dados especialmente padrões relativos à combinação fonotática do CCV diferentemente do observado em línguas como o Holandês e o Português Europeu. Os Quadros 3 e 4 abaixo descrevem, resumidamente, os estágios observados por Fikkert (1994) no Holandês e Freitas (1997) no PE, a fim de delinear uma comparação entre a aquisição do ataque ramificado em Português Brasileiro e em línguas em que o desenvolvimento estrutural CCV parece mais demarcado na fala infantil, como se nota a seguir. Ambos os estudos seguem metodologia naturalística, acompanhando 7 crianças portuguesas e 12 crianças holandesas.

\section{Estágios da aquisição do ataque ramificado em Holandês}

Estágio 0: CCV não surge como um alvo possivel

Estágio 1: CCV é reduzido ao seu primeiro elemento $\left(\mathrm{C}_{1} \mathrm{C}_{2} \rightarrow \mathrm{C}_{1} \varnothing\right)$

Estágio 2: $\mathrm{CCV}$ é reduzido ao seu segundo elemento $\left(\mathrm{C}_{1} \mathrm{C}_{2} \rightarrow \emptyset \mathrm{C}_{2}\right)$

Estágio 3: as duas consoantes do $\mathrm{CCV}$ são produzidas $\left(\mathrm{C}_{1} \mathrm{C}_{2}\right)$

Quadro 3: Estágios da aquisição CCV em Holandês (adaptado de Fikkert, 1994)

\section{Estágios da aquisição do ataque ramificado em Português Europeu}

Estágio 0: $\mathrm{CCV}$ não surge como um alvo possivel

Estágio 1: CCV é reduzido ao seu primeiro elemento ou apagado $\left(\mathrm{C}_{1} \mathrm{C}_{2} \rightarrow \mathrm{C}_{1} \varnothing\right.$ ou $\left.\mathrm{C}_{1} \mathrm{C}_{2} \rightarrow \emptyset \varnothing\right)$

Estágio 2: CCV é reduzido ao seu segundo elemento $\left(\mathrm{C}_{1} \mathrm{C}_{2} \rightarrow \varnothing \mathrm{C}_{2}\right)$

Estágio 3: as duas consoantes do $\mathrm{CCV}$ são produzidas $\left(\mathrm{C}_{1} \mathrm{C}_{2}\right)$

Estágio $3 a$ : como estando associadas a uma só posição no esqueleto $\left(\mathrm{C}_{1} \mathrm{C}_{2}\right)$

Estágio 3b: com vogal epentética ou conforme o alvo $\left(\mathrm{C}_{1} \mathrm{C}_{2} \rightarrow \mathrm{C}_{1} \mathrm{~V} \cdot \mathrm{C}_{2} \mathrm{~V}\right.$ ou $\left.\mathrm{C}_{1} \mathrm{C}_{2}\right)$

Estágio 3c: conforme o alvo $\left(\mathrm{C}_{1} \mathrm{C}_{2}\right)$

Quadro 4: Estágios da aquisição CCV em Português Europeu (adaptado de Freitas, 1997)

Tanto em Holandês quanto em PE, o Estágio 0 compõe-se por um momento de seleção lexical, estratégia definida como uma evitação deliberada da criança a determinados elementos linguísticos - período não 
reportado nos estudos em PB. Fikkert (1994) ressalta, contudo, que tanto a estratégia de seleção como a estratégia de redução $\mathrm{C}_{1} \mathrm{~V}$ representariam um mesmo momento no desenvolvimento infantil: a ausência da estrutura CCV no sistema fonológico da criança.

Acerca do Estágio 0, gostaríamos de destacar que, dada a metodologia naturalística utilizada, é possível cogitar que a seleção lexical não seria, de fato, uma estratégia deliberadamente aplicada pela criança a fim de evitar a produção de certos contextos, mas, sim, o resultado da própria ausência de palavras contendo CCV no vocabulário infantil - assim como observado nos contextos $\mathrm{C} / \mathrm{l} / \mathrm{V}$ em $\mathrm{PB}$.

Passando aos Estágios 1 e 2, Freitas (1997) aponta que, embora a estratégia de Redução a $\mathrm{C}_{1} \mathrm{~V}$ tenha se mostrado produtiva em $\mathrm{PE}$ - assim como a estratégia de apagamento de todo o ataque-, a estratégia de Redução a $C_{2} V$ observada por Fikkert (1994) como produtiva no Holandês (e também em Espanhol e em Alemão, segundo a autora), pouco se apresentou nos dados das crianças portuguesas. Em relação ao PB, tanto a estratégia de apagamento de todo o ataque como a redução $\mathrm{C}_{2} \mathrm{~V}$ são referidas como muito pouco frequentes pela literatura acima apresentada.

Relativamente ao Estágio 3, por fim, observa-se no corpus de Fikkert (1994) o completo desenvolvimento da sílaba CCV, e em Freitas (1997) a emergência das primeiras realizações-alvo do CCV. Em PE, o Estágio 3 se subdivide em três momentos, devido à proposta de Freitas (1997) de que estas primeiras realizações-alvo do CCV não devem, de fato, ser associadas ao desenvolvimento do molde ramificado na fala infantil, mas sim à consideração da sequência $\mathrm{C}_{1} \mathrm{C}_{2}$ como um segmento complexo, que ocuparia ainda uma única posição silábica no nó Ataque. Em seguida, o CCV sofreria epênteses - como em [bi'ru.fe] 'bruxa' ou [fí 'lors] 'flores' (FREITAS, 1997, p. 189) - que demarcariam a representação da estrutura $\mathrm{CCV}$ na Fonologia da criança.

Nota-se na literatura acima abordada que duas são as principais razões apontadas para justificar a incidência dos padrões observados ao desenvolvimento do ataque ramificado CCV na fala infantil, sejam estes padrões descritos como sistemáticos e regulares ou não:

i) tais padrões constituiriam uma tendência geral do percurso de aquisição fonológica;

ii) tais padrões dependeriam do desenvolvimento individual de cada criança, de suas preferências linguísticas e do input à sua disposição. 
Enquanto i) descreve os padrões observados na aquisição $\mathrm{CCV}$ como regulares, prevendo que "as crianças superam esses processos [i.e, estratégias de reparo] em épocas semelhantes e de maneira semelhante, num desenvolvimento regido por processos com características universais" (LAMPRECHT, 1993, p. 101), a descrição em ii) atribui a observação dos padrões ou de sua ausência a diferenças individuais, "ao lado das características universais (...). Assim, há diferenças não só na idade como no ritmo de superação dos processos. Também notam-se diferenças individuais através das estratégias adotadas pelas crianças para superar um processo" (LAMPRECHT, 1993, p. 101-102). Ainda segundo Lamprecht (1993, p. 102), relativamente aos percursos individuais, "na superação da redução de encontros consonantais verificou-se que os sujeitos empregaram estratégias diversas, até opostas, dando preferência a um determinado tipo de composição do encontro ou a uma determinada obstruinte ou líquida".

Conforme os resultados expostos nessa seção 2, ambos os padrões i) e ii) podem ser encontrados nos dados do PB. No presente artigo, gostaríamos de sugerir e investigar uma terceira possível razão: a metodologia. $\mathrm{Na}$ literatura apresentada, tanto foram utilizados métodos longitudinais como métodos transversais - estes últimos coletados com base em instrumentos fonoaudiológicos de elicitação -, ambos obtendo resultados distintos, e por vezes não homogêneos entre si (como, p. ex., Ávila (2000) e Ribas (2002), que encontraram tendências opostas em coleta transversal, assim como Staudt (2008) e Lamprecht (1993), em coleta longitudinal). Paralelamente, tem-se que as pesquisas em Português Europeu, Holandês e Francês, utilizando dados longitudinais, observaram estágios estruturais demarcados que, em PB, foram majoritariamente relatados em estudos de tipo transversal. Tendo estas divergências em vista, este artigo pretende contribuir à literatura ao buscar mensurar se parte das distinções observadas nos estudos acima pode ser atribuída a causas extrínsecas ao percurso de desenvolvimento infantil - especificamente, às formas de que o pesquisador dispõe para observar este percurso.

Deste modo, desejando examinar até que ponto i) tendências regulares gerais, ii) preferências individuais e iii) efeitos metodológicos podem influir sobre a observação do percurso de aquisição do ataque ramificado em PB, passamos a descrever os dados naturalísticos e experimentais aqui utilizados. 


\section{Novos dados à aquisição CCV: Metodologia na- turalística e experimental}

3.1 Materiais, métodos e procedimentos: Dados naturalísticos

O corpus naturalístico utilizado na pesquisa é composto por 203 gravações da fala de 3 crianças, Am., Lz. e Ar., duas meninas e um menino, pertencentes ao banco de dados do Projeto de Aquisição de Ritmo (SANTOS, 2005). Os sujeitos foram gravados quinzenalmente, em interação espontânea com seus familiares e cuidadores, nas suas próprias residências. $\mathrm{O}$ período analisado abrangeu desde as primeiras tentativas de produção do ataque ramificado CCV de cada sujeito até suas últimas sessões disponíveis no banco de dados, compreendendo as faixas etárias listadas no Quadro 5 abaixo:

\begin{tabular}{|l|}
\hline Lz. 1;7 - 5;6 anos, 92 gravações \\
Am. $2 ; 0-4 ; 0$ anos, 44 gravações \\
Ar. 1;8 - 4;11 anos, 67 gravações \\
\hline
\end{tabular}

Quadro 5: Idade e quantidade de gravações analisadas por criança - Corpus naturalistico

Todas as produções infantis contendo sílabas de ataque ramificado CCV (oclusiva+líquida) foram selecionadas com o auxílio de um script computacional e analisadas acusticamente por meio do software Praat. Tal análise foi conduzida a fim de levar em consideração possíveis contrastes encobertos - isto é, diferenciações produzidas pela criança (nos segmentos, na duração ou na frequência dos formantes, por exemplo), mas não percebidas oitivamente pelo adulto (cf. Scobbie, 1998; Berti, 2006; Berti \& Marino, 2011).

3.2 Materiais, métodos e procedimentos: Dados experimentais

O corpus experimental, por sua vez, compõe-se por dados de 49 crianças entre $2 ; 4$ e 5;10 anos (24 meninas e 25 meninos), que frequentavam os Módulos 2 ou 3 de uma creche localizada em São Paulo - SP (aprovado pelo Comitê de Ética em Pesquisa sob o CAAE 43086214.4.0000.5498). O experimento consistiu na realização de tarefas de repetição de palavras contendo contextos de ataque ramificado $\mathrm{CCV}$, em que $\mathrm{C}_{1}$ se compunha pelas plosivas /p, t, k, b, d, g/, $\mathrm{C}_{2}$ pelas líquidas /l, $\mathrm{r} /$ e V pelas vogais /a/, /i/ e /o, u/. Um total de 52 alvos CCV foram apresentados às crianças, como exemplificado no Quadro 6: 


\begin{tabular}{|ll|}
\hline prato & placa \\
bruxa & blusa \\
trilho & $\underline{\text { Tlico }}$ \\
Drica & $\underline{\text { Dlico }}$ \\
$\underline{\text { Craca }}$ & classe \\
grupo & Glub \\
\hline
\end{tabular}

Quadro 6: Exemplos de palavras utilizadas no estudo experimental. Pseudopalavras sublinhadas

Conforme observado no Quadro 6, os alvos compunham-se de nomes comuns ao vocabulário infantil (objetos, animais, personagens, etc.) e nomes inventados (pseudopalavras). O uso de pseudopalavras deu-se para atender à produção de contextos segmentais raros ou ausentes na língua, como /tl, dl/, e também à produção de contextos em que não foi possível encontrar itens lexicais passíveis de ilustração pertencentes ao léxico infantil. Todas as palavras selecionadas apresentavam molde prosódico do tipo /'CCV.CV/, com as sílabas-alvo localizadas na posição tônica da palavra.

\subsection{Classificação das produções infantis: Estratégias de reparo}

O Quadro 7 abaixo categoriza, descreve e exemplifica os reparos à aquisição da sílaba CCV observados nos contextos analisados, também referidos na literatura por Teixeira (1988), Lamprecht (1993), Ribas (2002) e Baesso (2009). Tais estratégias foram também atestadas por Greenlee (1974) em outras seis línguas: inglês, francês, estoniano, sérvio, esloveno e tcheco.

\begin{tabular}{|c|c|c|}
\hline Estratégias de reparo & Descrição & Exemplo \\
\hline Apagamento do $\mathrm{CCV}$ & Apagamento da silaba contendo $\mathrm{CCV}$ & 'floresta' ['عs.te] \\
\hline Apagamento de $C_{1}+C_{2}$ & Produção apenas da vogal do $\mathrm{CCV}$ & 'classe' ['a.sI] \\
\hline Apagamento de $C_{1}$ & Produção dos segmentos em $\mathrm{C}_{2} \mathrm{~V}$ & 'Drica' ['li.ke] ${ }^{2 a}$ \\
\hline Apagamento de $\mathrm{C}_{2}$ & Produção dos segmentos em $\mathrm{C}_{1} \mathrm{~V}$ & 'bruxa' ['bu.se] \\
\hline Substituição de $C_{1}$ & $\begin{array}{l}\text { Alteração de ponto de articulação, modo ou } \\
\text { vozeamento do segmento em } \mathrm{C}_{1}\end{array}$ & $\begin{array}{l}\text { 'trave' ['kra.vi] } \\
\text { 'Dlopo' ['go.pu] }\end{array}$ \\
\hline Substituição de $C_{2}$ & Alteração da liquida em $\mathrm{C}_{2}$ & $\begin{array}{l}\text { 'Drica' ['dli.ke] } \\
\text { 'classe' ['kra.sI] }\end{array}$ \\
\hline Metátese & Alteração da posição $\mathrm{C}_{1} \mathrm{C}_{2}: \mathrm{CCV} \gg \mathrm{CVC}$ & $\begin{array}{l}\text { 'cobra' ['kor.be] } \\
\text { 'Pluto' ['pul.tv] }\end{array}$ \\
\hline Transposição & Movimento da liquida à silaba adjacente & 'pedra' ['pre.de] \\
\hline Movimento recíproco & Permuta entre segmentos de duas silabas adjacentes & $\begin{array}{l}\text { 'dragão' [gra'dãw] } \\
\text { 'Tlico' ['kli.tu] }\end{array}$ \\
\hline Coalescência & Assimilação de [+cont] em $\mathrm{C}_{2}$ por $\mathrm{C}_{1}$ & 'Pluto' ['fu.tu] \\
\hline Epêntese vocálica & Adição de uma vogal entre $\mathrm{C}_{1}$ e $\mathrm{C}_{2}$ & $\begin{array}{l}\text { 'troco' [to'ro.kv] } \\
\text { 'Tlibo' ['tfli.bu] }\end{array}$ \\
\hline
\end{tabular}

Quadro 7: Estratégias de reparo categorizadas na pesquisa² 
Ressaltamos que, como o Apagamento $\mathrm{C}_{2}$ foi apontado pela literatura descrita na seção 2 como a mais frequente estratégia de reparo ao ataque ramificado $\mathrm{CCV}$, optamos por classificar esta estratégia em uma categoria própria na pesquisa. Deste modo, as produções infantis foram categorizadas em: Produção-alvo (quando CCV foi produzido do mesmo modo que um falante adulto); Apagamento $C C V>C_{1} V$ (em que houve supressão do segmento líquido em $\mathrm{C}_{2}$ ) e Outros reparos (em que qualquer outra das estratégias listadas no Quadro 7 foi imposta ao estímulo-alvo pela criança).

\subsection{Organização dos dados: Faixas de Produção CCV versus Faixas etárias}

Para promover uma comparação apropriada dos dados, a fim de identificar semelhanças e diferenças tanto interfalantes - nos dados experimentais -, quanto intrafalantes - nas diversas sessões naturalísticas de cada sujeito -, optamos por designar como critério à divisão dos dados infantis não a idade ou faixa etária, mas sim o percentual de realizações-alvo do ataque ramificado - critério também utilizado em instrumentos fonoaudiológicos como o teste PCC - Porcentagem de Consoantes Corretas. Tal divisão se fez necessária devido à observação de que crianças de uma mesma faixa etária apresentavam taxas de realização-alvo do CCV bastante distintas. Enquanto o fator Idade admitiria a comparação de diferentes momentos de desenvolvimento silábico desde que estes fossem referentes a crianças de uma mesma idade, o percentual de produções-alvo CCV viabiliza a comparação entre crianças de mesmos momentos de desenvolvimento silábico. Desta forma, os dados da pesquisa foram divididos em cinco grupos de desenvolvimento da ramificação de ataque, em que 1 corresponde à ausência (ou reprodução mecânica e esporádica) do $\mathrm{CCV}$ e 5 corresponde à aquisição do CCV, conforme apresentado no Quadro 8 abaixo. ${ }^{3}$

\begin{tabular}{c|l|l|l|} 
Divisão & Realizações-alvo & \multicolumn{1}{|c|}{ Experimental } & \multicolumn{1}{c|}{ Naturalístico } \\
\hline Grupo 1 & $0 \%$ a $5 \%$ & 15 crianças & Lz 1;7-3;1 anos; Am 2;0-4;0 anos; Ar 1;8-4;11 anos \\
\hline Grupo 2 & $6 \%$ a $40 \%$ & 7 crianças & Lz 3;01-4;08 anos \\
\hline Grupo 3 & $41 \%$ a $60 \%$ & 5 crianças & $\mathrm{Lz} 4 ; 09-5 ; 03$ anos \\
\hline Grupo 4 & $61 \%$ a $75 \%$ & 13 crianças & Lz 5;03-5;06 anos \\
\hline Grupo 5 & Acima de $76 \%$ & 9 crianças & - \\
\hline
\end{tabular}

Quadro 8: Divisão das produções infantis conforme o percentual de realizações-alvo CCV 


\section{Resultados naturalísticos e experimentais: apre- sentação e cotejo à literatura}

Nesta seção, os resultados obtidos por meio das coletas naturalística e experimental serão apresentados, visando observar se padrões semelhantes ou distintos àqueles descritos na literatura podem também ser encontrados em nossos dados, assim como observar, nos casos de divergência, quais padrões podem ser corroborados pelos presentes resultados, e se a própria metodologia de coleta pode apontar para tendências distintas.

Trazemos na Tabela 1 abaixo a distribuição geral das produções infantis: se realizada como na forma alvo; se reduzida a $\mathrm{C}_{1} \mathrm{~V}$ por meio do apagamento de $\mathrm{C}_{2}$ ou se modificada por meio de outras estratégias de reparo.

\begin{tabular}{|c|c|c|c|c|c|c|c|}
\hline \multicolumn{8}{|c|}{$\%$ Naturalístico } \\
\hline Informante & \multicolumn{5}{|l|}{$\mathrm{Lz}$} & \multirow{2}{*}{\begin{tabular}{|l|} 
Am \\
Gl (714)
\end{tabular}} & \multirow{2}{*}{\begin{tabular}{|l|} 
Ar \\
Gl (1510)
\end{tabular}} \\
\hline Grupos & Gl (365) & G2 (1085) & G3 (431) & G4 & 211) & & \\
\hline Produção-alvo & 6,03 & 28,76 & 51,51 & 66,3 & & 1,82 & 1,52 \\
\hline $\mathrm{CCV}>\mathrm{C}_{1} \mathrm{~V}$ & 90,41 & 66,54 & 44,78 & 27,9 & & 89,78 & 99,56 \\
\hline Outros reparos & 3,56 & 4,7 & 3,71 & 5,69 & & 8,4 & 1,92 \\
\hline \multicolumn{8}{|c|}{$\%$ Experimental } \\
\hline Grupos & Gl (884) & G2 (441 & G3 (3. & & G4 & (824) & G5 (585) \\
\hline Produção-alvo & 0,79 & 28,34 & 49,7 & & 63,7 & & 75,38 \\
\hline $\mathrm{CCV}>\mathrm{C}_{1} \mathrm{~V}$ & 70,59 & 26,76 & 5,79 & & 10,8 & & 6,33 \\
\hline Outros reparos & 28,62 & 44,9 & 44,51 & & 25,4 & & 18,29 \\
\hline
\end{tabular}

Tabela 1: Distribuição geral das produções CCV infantis

Observa-se na Tabela 1 que a estratégia de Apagamento $\mathrm{C}_{2}(\mathrm{CCV}>$ $\mathrm{C}_{1} \mathrm{~V}$ ) apresenta-se como o reparo mais utilizado nos dados naturalísticos, tanto no período inicial da fala dos três sujeitos, Lz, Am e Ar, como ao longo dos grupos de desenvolvimento G1 a G4 de Lz. Já no estudo experimental a estratégia $\mathrm{CCV}>\mathrm{C}_{1} \mathrm{~V}$ tem aplicação predominante somente em G1, prevalecendo a aplicação das estratégias da categoria Outros reparos a partir de G2, contrariamente ao descrito pela literatura referida anteriormente.

Na Tabela 2 a seguir discriminam-se os percentuais de aplicação das diferentes modificações silábicas classificadas na categoria Outros reparos. Entre parênteses, tem-se o total de reparos recolhidos em cada grupo. 


\begin{tabular}{|c|c|c|c|c|c|c|c|c|c|}
\hline \multicolumn{10}{|c|}{ \% Naturalístico } \\
\hline \multirow{2}{*}{ Reparos } & \multicolumn{6}{|c|}{$\mathrm{Lz}$} & \multicolumn{2}{|c|}{$\mathrm{Am}$} & \\
\hline & Gl (14) & G2 (54) & \multicolumn{2}{|c|}{ G3 (16) } & \multicolumn{2}{|c|}{ G4 (14) } & Gl (60) & & \\
\hline Apagamento $\mathrm{C}_{1}$ & 7,14 & 1,85 & \multicolumn{2}{|c|}{-} & \multicolumn{2}{|c|}{-} & \multicolumn{2}{|c|}{-} & - \\
\hline Apagamento $\mathrm{C}_{1} \mathrm{C}_{2}$ & 7,14 & - & \multicolumn{2}{|c|}{-} & \multicolumn{2}{|c|}{ - } & \multicolumn{2}{|l|}{-} & - \\
\hline Apagamento $\mathrm{CCV}$ & - & - & \multicolumn{2}{|c|}{-} & \multicolumn{2}{|c|}{ - } & \multicolumn{2}{|c|}{-} & - \\
\hline Coalescência & - & - & \multicolumn{2}{|c|}{6,25} & \multicolumn{2}{|c|}{-} & \multicolumn{2}{|c|}{5} & - \\
\hline Epêntese de vogal & 7,14 & 20,37 & \multicolumn{2}{|c|}{62,5} & \multicolumn{2}{|c|}{50} & \multicolumn{2}{|c|}{3,33} & - \\
\hline Inserção de consoante & 7,14 & 3,7 & \multicolumn{2}{|c|}{6,25} & 7, & 14 & - & & - \\
\hline Movimento Reciproco & - & - & & 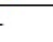 & 7, & 14 & 1,6 & & 3,57 \\
\hline Metátese & - & 3,7 & & & & - & - & & 7,14 \\
\hline Substituição $C_{1}$ & 57,14 & 9,26 & & . & 7, & 14 & $78, ?$ & & 60,71 \\
\hline Substituição $\mathrm{C}_{2}$ & 14,29 & 46,3 & & 5 & 28 &, 57 & 11, & & 28,57 \\
\hline Transposição & - & 14,81 & & - & 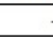 & - & - & & - \\
\hline & & $\%$ Experi & uent & & & & & & \\
\hline Reparos & G1 (308 & G2 & 267) & G3 & 184) & G4 & (241) & & $5(123)$ \\
\hline Apagamento $\mathrm{C}_{1}$ & 5,84 & & & & & & 98 & & 10,57 \\
\hline Apagamento $\mathrm{C}_{1} \mathrm{C}_{2}$ & 4,87 & & & & & & 83 & & 0,81 \\
\hline Apagamento $\mathrm{CCV}$ & - & & & & & & - & & - \\
\hline Coalescência & 0,97 & & & & & & - & & 0,81 \\
\hline Epêntese de vogal & 0,32 & & & & & & 73 & & 12,2 \\
\hline Inserção de consoante & - & & & & & & 41 & & - \\
\hline Movimento Reciproco & 3,25 & & & & & & 41 & & 1,63 \\
\hline Metátese & 0,65 & & & & & & 41 & & - \\
\hline Substituição $\mathrm{C}_{1}$ & 74,68 & & & & & & 08 & & 55,28 \\
\hline Substituição $\mathrm{C}_{2}$ & 8,77 & & & & & & 99 & & 18,7 \\
\hline Transposição & 0,65 & & & & & & 15 & & - \\
\hline
\end{tabular}

Tabela 2: Distribuição das estratégias classificadas em Outros reparos

Observa-se na Tabela 2 variação no emprego das estratégias a depender do Grupo em que são aplicadas, o que pode indicar uma preferência por reparos distintos associada ao momento do desenvolvimento silábico da criança.Tanto no estudo naturalístico quanto no experimental, tem-se maior emprego das estratégias de Substituição $C_{1}$ no grupo G1, ocorrência que pode relacionar-se à própria aquisição dos segmentos oclusivos por parte dos sujeitos, conforme Ribas (2002); em G2 tem-se, por sua vez, maior proporção de reparos abrangendo a substituição da líquida em posição $\mathrm{C}_{2}$, além de um crescimento na aplicação da Epêntese e, na fala de Lz, também da Transposição. Em G3e G4 as estratégias de Epêntese e Substituição $\mathrm{C}_{2}$ mantém alta proporção, com prevalência da primeira no estudo naturalístico e da segunda no estudo experimental, além da estratégia de Substituição $C_{1}$ neste último, também observada em 
G5, e que se relaciona à produção dos alvos /tl, dl/, majoritariamente.

Para examinar as preferências segmentais à posição $\mathrm{C}_{2}$ do $\mathrm{CCV}$, trazemos a Tabela 3 a seguir, que contém a direção de aplicação da estratégia de Substituição $\mathrm{C}_{2}$, em dados absolutos:

\begin{tabular}{|c|c|c|c|c|c|c|}
\hline \multirow{2}{*}{ Naturalístico } & \multicolumn{4}{|c|}{ Lz } & \multirow{2}{*}{$\begin{array}{c}\text { Am } \\
\text { Gl (7) }\end{array}$} & \multirow{2}{*}{$\begin{array}{c}\text { Ar } \\
\text { Gl (8) }\end{array}$} \\
\hline & Gl (2) & G2 (25) & G3 (4) & G4 (4) & & \\
\hline$\Lambda \rightarrow / 5 /$ & 1 & 0 & 0 & 0 & 0 & 0 \\
\hline$\Lambda \rightarrow$ glide/outros & 0 & 0 & 0 & 0 & 0 & 0 \\
\hline$/ \mathrm{s} / \rightarrow N$ & 0 & 18 & 4 & 4 & 6 & 0 \\
\hline$/ \mathrm{s} / \rightarrow$ glide/outros & 1 & 7 & 0 & 0 & 1 & 8 \\
\hline Experimental & Gl (27) & \multicolumn{2}{|c|}{ G2 (108) } & G3 (71) & G4 (53) & G5 (23) \\
\hline$\Lambda \rightarrow / \mathrm{s}$ & 3 & \multicolumn{2}{|c|}{51} & 58 & 34 & 15 \\
\hline $\mathrm{N} \rightarrow$ glide/outros & 13 & \multicolumn{2}{|c|}{5} & 2 & 0 & 4 \\
\hline$/ \mathrm{s} / \rightarrow / /$ & 7 & \multicolumn{2}{|c|}{50} & 4 & 16 & 4 \\
\hline$/ \mathrm{s} / \rightarrow$ glide/outros & 4 & \multicolumn{2}{|c|}{2} & 7 & 3 & 0 \\
\hline
\end{tabular}

Tabela 3: Direção de aplicação da estratégia de Substituição $C_{2}$

Diferentes direções de substituição são observadas nos estudos naturalístico e experimental na Tabela 3: enquanto a fala espontânea tende a substituir a líquida não-lateral /r/pela líquida lateral ou por outros segmentos soantes (majoritariamente [j]), tal tendência não se mantém no estudo experimental, em que se nota também a líquida lateral como um alvo recorrente às modificações em $\mathrm{C}_{2}$. Embora a proporção entre as modificações visando à líquida lateral ou não-lateral se apresente equilibrada em G1 e G2 na coleta experimental, a partir de G3 as sílabas C/1/V apresentam-se como o principal alvo às Substituições $\mathrm{C}_{2}$. Tal padrão corrobora os resultados de Teixeira (1988), Lamprecht (1993) e Galea (2008) referidos anteriormente.É necessário salientar, contudo, que nos dados naturalísticos somente uma única ocorrência da estratégia de Substituição $\mathrm{C}_{2}$ aplicou-se em contexto C/l/V,'Mogli' ['mo.gi.ri](Lz 2;04 anos), dada a baixa frequência deste grupo silábico na fala infantil e mesmo na língua-alvo (Totais de alvos contendo CCV produzidos pelos sujeitos: Am: C/r/V: 731; C/1/V: 43; Ar: C/r/V:1405; C/1/V: 65;Lz: C/r/V:2194; C/1/V: 120), diferentemente do estudo experimental, em que a quantidade de estímulos $\mathrm{C} / \mathrm{l} / \mathrm{V}$ e $\mathrm{C} / \mathrm{r} / \mathrm{V}$ foi balanceada. Faz-se possível, assim, atribuir à própria distribuição dos dados em cada estudo a diferença observada entre a proporção de substituições /1/ e /r/.

Ainda tratando da Substituição $C_{2}$, observou-se que, no estudo experimental, 2 crianças apresentaram substituições sistemáticas da líquida, 
em direção $\mathrm{C} / \mathrm{l} / \mathrm{V}>>\mathrm{C} / \mathrm{r} / \mathrm{V}$, conforme observado nos exemplos em (2). Outros sujeitos, por outro lado, aplicaram o processo em ambas as direções, mesmo em contextos semelhantes, conforme observado em (3):

(2) (Informante 11, G2, 3;3 anos)

['kru.br] 'clube'

(Informante 26, G3, 4;1 anos)

['pra.ke] 'placa'

['bru.ze] 'blusa'

['tro.pu] 'Tlopo'

['kra.sI] 'classe'

['pru.tv] 'Pluto'

['dro.pu] 'Dlopo'

['pru.tv] 'Pluto'

(3) (Informante 24, G4, 4; 10 anos)

(Informante 32, G4, 5;3 anos)

['dri.bu] 'Dribo'

['tri.ku] 'Tlico'

['vli.ku] 'Drigo'

['tri.bu] 'Tlibo'

['kri.kI] "clique'

['kli.pr] "clipe'

['pli.ti] 'Pritt'

['plike] 'Plica'

A fim de observar se a líquida lateral /1/ ou o tepe / $/$ / poderiam desencadear diferentes proporções de aplicação de estratégias de reparo, complementando as tendências observadas na Tabela 3, o teste estatístico $t$ de Student foi aplicado aos resultados experimentais da pesquisa na categoria Outros reparos. Embora nem todos os Grupos tenham apresentado significância estatística ao tipo de líquida, nos Grupos em que diferenças significativas foram constatadas (G1, G3, G5) a líquida lateral mostrou-se o alvo preferencial à aplicação de estratégias em comparação à líquida não-lateral, mesmo padrão observado a partir de G3 no estudo experimental.

Para verificar longitudinalmente o desenvolvimento dos sujeitos do estudo naturalístico, por fim, trazemos os Gráficos 1-3 abaixo, que resumem o tipo de realização dos alvos CCV articuladas pelas crianças ${ }^{4}$. Observa-se nas plotagens que, embora acompanhados até 4;0, 4;11 e 5;6 anos, respectivamente, nenhum dos sujeitos da pesquisa adquiriu o ataque ramificado, considerando-se como critério à aquisição o mínimo de $75 \%$ de produções-alvo em 3 sessões consecutivas. Enquanto Am e Ar permaneceram no Grupo 1 durante todo o período analisado, Lz pode ser acompanhada até o Grupo 4. Note-se que, aos 4;0 anos, Lz já se encontrava no Grupo 2 (diferentemente de Am), enquanto aos 4;11 anos, no Grupo 3 (diferentemente de Ar). Tal observação corrobora o indicado por Lamprecht (1993) acerca da variabilidade na idade.

Note-se nos Gráficos 1-3, também, que tal como descrito na Tabela 1 anteriormente, o emprego de estratégias de reparo no estudo naturalísti- 
co apresentou baixos percentuais de aplicação, menos de $10 \%$ dos dados para cada sujeito, padrão semelhante ao encontrado no estudo transversal de Ribas (2002) e no longitudinal de Staudt (2008).
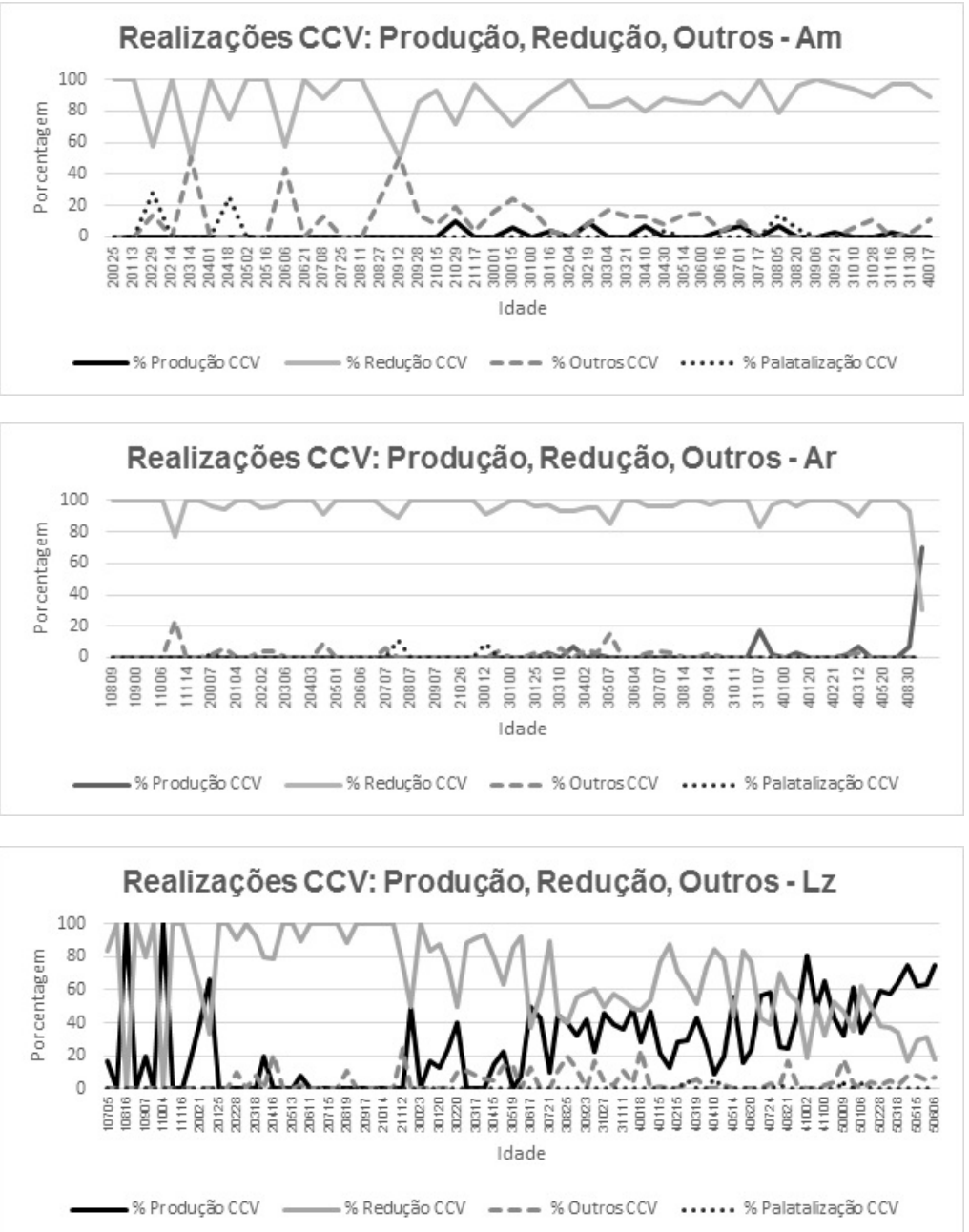

Gráfico 3: Realizações CCV de Lz

Devido à baixa proporção de reparos diferentes da redução $\mathrm{C}_{1} \mathrm{~V}$, não são observados estágios intermediários em que o emprego de deter- 
minadas estratégias é utilizado para superar o apagamento $\mathrm{C}_{2}$ na produção $\mathrm{CCV}$ - diferentemente do observado no Holandês, no Português Europeu e no Francês, por exemplo. Adicione-se a isto, ademais, os dados da Tabela 2 , em que se percebe que a aplicação de estratégias de reparo na fala de cada sujeito da amostra não é sistemática, isto é, há uso de diversos reparos em concomitância, e não o emprego de uma única estratégia específica como forma de adaptação input-output. Observe-se, como ilustração, as produções [gur'dej], [gu.ru'dej], [gu.de'rej] e [glu'da.dv], referentes ao radical verbal 'grudar', recolhidas na sessão 4;08 da fala de Lz (G2).

Dada a observação de que nenhum dos sujeitos Am, Ar e Lz completou o percurso de aquisição $\mathrm{CCV}$, passamos a examinar o período de surgimento das diferentes combinações fonotáticas que podem compor a estrutura de ataque ramificado, trazidas na Tabela 4 a seguir:

\begin{tabular}{|c|c|c|c|c|c|c|c|}
\hline \multicolumn{2}{|c|}{} & $/ \mathbf{p} /$ & $/ \mathbf{b} /$ & $/ \mathbf{t} /$ & $/ \mathbf{d} /$ & $/ \mathbf{k} /$ & $/ \mathbf{g} /$ \\
\hline \multirow{2}{*}{$\mathbf{L z}$} & $/ \mathbf{r} /$ & $2 ; 06$ & $1 ; 07$ & $3 ; 01$ & $4 ; 05$ & $3 ; 10$ & $3 ; 06$ \\
\cline { 2 - 8 } & $\mathbf{I} /$ & $2 ; 06$ & $4 ; 06$ & - & - & $4 ; 06$ & $5 ; 03$ \\
\hline \multirow{2}{*}{$\mathbf{A m}$} & $/ \mathbf{r} /$ & $3 ; 07$ & $2 ; 10$ & $3 ; 11$ & - & - & - \\
\cline { 2 - 8 } & $\mathbf{I} /$ & - & - & - & - & $3 ; 04$ & - \\
\hline \multirow{2}{*}{$\mathbf{*} A \mathbf{r}$} & $/ \mathbf{r} /$ & $3 ; 03$ & $3 ; 02$ & $4 ; 01$ & $3 ; 04$ & $4 ; 11$ & - \\
\cline { 2 - 8 } & $/ \mathbf{I}$ & $4 ; 03$ & $3 ; 11$ & - & - & $4 ; 03$ & - \\
\hline
\end{tabular}

Tabela 4: Idade de surgimento das combinações fonotáticas CCV na fala de Lz, Am e Ar. 0 símbolo (-) indica a ausência de produções corretas de uma dada combinação fonotática

Embora a maior parte das combinações fonotáticas CCV tenha surgido até $\mathrm{G} 2$ na fala dos informantes (à exceção de /gl/ em Lz, surgida em G4), tal emergência não foi concomitante quando se analisam, propriamente, as idades de seu surgimento. Não foi possível, contudo, verificar uma ordenação regular na emergência destas combinações $\mathrm{C}_{1} \mathrm{C}_{2}$ no corpus: observou-se que as oclusivas em posição $C_{1}$ não surgem na fala dos informantes segundo classes naturais, por exemplo; comparando os dados de Am, Ar e Lz, mesmo a ordem de emergência dos segmentos individualmente não se mostrou regular. $\mathrm{O}$ mesmo pôde ser verificado com referência às líquidas em posição $\mathrm{C}_{2}$ : embora as combinações com / $/$ tenham sido majoritariamente produzidas antes de $/ 1 /$, as sequências / $\mathrm{pl} /$ na fala de Lz e / $\mathrm{kl} /$ na fala de Am e Ar surgiram antes de suas contrapartes $/ \mathrm{pr} /, / \mathrm{kr} /$, não sendo possível observar um surgimento categórico de combinações com tepe perante combinações com lateral, por exem- 
plo. Observou-se, ainda, que nenhum par $\mathrm{C}_{1} / 1 / \mathrm{V}$ e $\mathrm{C}_{1} / \mathrm{r} / \mathrm{V}$ teve emergência numa mesma sessão, na fala das três crianças. Na fala de Am, nenhuma das combinações CCV apresenta sua contraparte líquida, isto é, em suas produções nenhuma oclusiva foi combinada com ambas as líquidas /1/ e / $/$ / até o término de seus dados, aos 4;0 anos. Já na fala de Ar e Lz, todas as oclusivas produzidas apresentaram combinações com ambas as líquidas lateral e não-lateral. Uma diferença mínima de 9 meses, no entanto, foi observada entre o surgimento das combinações com uma ou outra líquida em suas produções. No desenvolvimento silábico de Lz, somente /kr, kl/ e /pr, pl/ foram combinações surgidas num mesmo grupo, G2; por outro lado, /br/, a primeira sílaba CCV a emergir nos dados de Lz, só teve sua contraparte /bl/ produzida a partir de G2; e /gr/, por fim, emergiu em G2 enquanto /gl/, em G4.

Tomando como base os gráficos, tabelas e exemplos apresentados nesta seção, observa-se que a distribuição e a frequência das estratégias de reparo, tanto nos contextos naturalísticos quanto experimentais, não parece evidenciar um percurso de desenvolvimento silábico dividido em estágios definidos - estágios que deveriam apresentar, segundo Teixeira (1988) e Lamprecht (1993), um uso regular e/ou sistemático de determinadas modificações silábicas de acordo com o grau de desenvolvimento da ramificação e de suas combinações segmentais. Deste modo, nossos resultados corroboram os estudos de Ribas (2002) e Staudt (2008), acerca da ausência de estágios estruturais no percurso CCV, e também Lamprecht (1993), Galea (2008) e, parcialmente, Teixeira (1988), acerca da dupla direcionalidade das modificações segmentais na posição $\mathrm{C}_{2}$.

5 Tendência geral, preferência individual, efeito metodológico: fatores de influência no percurso de aquisição do ataque ramificado

Como descrito na seção anterior, o percurso de aquisição do ataque ramificado em PB, diferentemente do Português Europeu, do Holandês e do Francês, não parece ocorrer segundo estágios intermediários regulares, sistemáticos e demarcados na fala infantil, tanto em relação à aquisição do molde silábico CCV como a suas combinações fonotáticas.

Embora a presença de estratégias de reparo seja mais recorrente no estudo experimental, o que poderia indicar a presença de estágios, destacamos que não parece haver uma estratégia específica regularmente aplicada pelos 
49 sujeitos analisados, de forma sistemática, sendo possível, inclusive, que contextos semelhantes apresentem diversas formas de adaptação input-output na produção de uma mesma criança. Verifica-se que os sujeitos seguem diferentes "trajetos" a fim de produzir a sílaba CCV como na sua forma-alvo: alguns, por exemplo, traçam um percurso Redução $C_{1} V>>$ Produção-alvo, partindo de um momento em que há o apagamento sistemático de $\mathrm{C}_{2}-$ atribuído à ausência do tipo CCV no sistema fonológico da criança - diretamente à articulação plena do ataque ramificado; outros sujeitos optam por modificar a lateralidade das líquidas em posição $\mathrm{C}_{2}$ antes de produzi-las conforme o alvo, como nos exemplos em (2) e (3); outros, ainda, aplicam a estratégia de epêntese, reparo que apresentou percentual de aplicação considerável na Tabela 2; e até mesmo as estratégias de metátese ou transposição, que são reportadas por Teixeira (1988) como frequentes embora pouco aplicadas em nossos dados. É notável, contudo, que estes são alguns dos muitos "trajetos" que a criança poderia tomar ao longo de seu percurso de aquisição silábica - veja-se, por exemplo, a variedade de outras estratégias recolhidas no Quadro 7, como a produção $\mathrm{C}_{2} \mathrm{~V}$, comum no Holandês, mas não em $\mathrm{PB}$ e $\mathrm{PE}$.

Deste modo, embora seja notório que cada criança parece traçar seus próprios "trajetos" - nem sempre regulares ou unidirecionais - no percurso de aquisição $\mathrm{CCV}$, parece razoável sugerir que, dado que estes percursos individuais frequentemente tomam os mesmos "trajetos", em detrimento da grande variedade de estratégias de reparo disponível à criança, é possível que as tendências gerais assinaladas por Teixeira (1988) e Lamprecht (1993) gerem uma gama controlada ou limitada de "trajetos" a serem cursados pelas crianças em seu percurso de aquisição silábica, "trajetos" que podem ser resultantes da própria organização da gramática infantil, do seu sistema linguístico-alvo ou mesmo da combinação de ambos.

Tentando responder, por fim, o motivo pelo qual se podem observar divergências entre o percurso de aquisição do ataque ramificado no Português Brasileiro quando comparado ao Português Europeu, ao Holandês e ao Francês - e mesmo quando comparado em diferentes estudos abordando o PB, propriamente -, três possíveis razões foram cogitadas pela presente pesquisa: i) tendências gerais do desenvolvimento fonológico infantil; ii) preferências individuais da criança - razões que parecem correlacionar-se; e iii) efeitos metodológicos relativos ao tipo de coleta de dados. Quanto à influência desta última, observa-se que parece haver circunstâncias em que o método de pesquisa pode interferir na observação da aquisição $\mathrm{CCV}$, especificamente, na delimitação de estágios compreendendo a estratégia de seleção lexical; e na 
demarcação da direção preferencial / / > /1/ na estratégia de Substituição $\mathrm{C}_{2}$, ambas relativas a coletas de fala espontânea. Tal interferência dá-se em virtude do surgimento não-controlado dos contextos-alvo enfocados pelo pesquisador, que depende da frequência de tais contextos na língua-alvo. No caso do ataque ramificado, somente cerca de 5\% das palavras em PB contêm sílabas CCV, e talvez um número ainda menor seja observável no vocabulário infantil. Dentre estas, por sua vez, majoritariamente observam-se contextos $\mathrm{C} / \mathrm{r} / \mathrm{V}$. Por este motivo, é possível que a seleção lexical ou os alvos e direção das substituições $\mathrm{C}_{2}$ se devam não à gramática em desenvolvimento da criança, mas sim às propriedades de sua língua-alvo. Outras interferências metodológicas não parecem, contudo, agir sobre o estudo do ataque ramificado CCV, uma vez que resultados semelhantes puderam ser observados em metodologias distintas.

\section{RECALLING THE CCV'S BRANCHING ONSETS ACQUISI- TION: STAGES OF SYLLABIC DEVELOPMENT}

\section{ABSTRACT}

This article aims to debate about the development stages in the course of acquisition of CCV branching onset syllables (Consonant1 + Consonant 2 + Vowel) - structure present in Brazilian Portuguese words like 'bruxa' witch, 'blusa' blouse, 'prato' plate, 'placa' plaque. Reviewing researches on Brazilian Portuguese as Teixeira (1988), Lamprecht (1993), Ávila (2000), Ribas (2002), Staudt (2008), Baesso (2009), this article examines possible motivations for the (non)observation of linguistic development stages in the $\mathrm{CCV}$ acquisition by Brazilian children. It is investigated whether such motivations may lie i) a general trend of the phonological acquisition path - as observed in the acquisition of other syllabic structures, like CVC coda; (ii) in the individual development of the subjects; or iii) in methodological effects related to the type of data collection. To verify these motivations, the study analyzes naturalistic data of 3 subjects, recorded biweekly, and experimental data of 49 subjects, recorded in a repetition test of words and pseudowords. The results indicate that the naturalistic data collection can affect the observation of syllabic development stages, since the unbalance of CCV targets and their phonotatic 
combination may affect the determination of strategies such as lexical selection and the origin/direction of substitutions in $\mathrm{CCV}$. Individual development was observed as correlated with the general phonological tendencies pointed out by Teixeira (1988) and Lamprecht (1993), because these generalizations may act as limits to the different paths possible to the child in CCV acquisition. The study also suggests that the type of strategy used to repair CCV also depends on the child's syllabic development.

KEYWORDS: Phonology; Language acquisition; Development stages; Syllable; Branching onsets

\section{NOTAS}

${ }^{1}$ Estratégias de reparo: aquilo "que as crianças realizam em lugar do segmento e/ ou da estrutura silábica que ainda não conhecem ou cuja produção não dominam” (Lamprecht, 2004, p. 28).

${ }^{2}$ a,b Conforme observa-se em ['li.ke] 'Drica' ou ['go.pv] 'Dlopo', uma mesma palavra pode sofrer simultaneamente mais de uma estratégia de reparo. Nestes casos, cada reparo foi contado como uma entrada.

${ }^{3}$ Para visualizar a idade e os percentuais de realização-alvo de cada criança, cf. Toni (2016). Os percentuais não consideraram a produção (correta ou não) de /tl, dl/.

${ }^{4}$ Este artigo apresenta parte dos resultados da dissertação de mestrado de Toni (2016). Neste estudo, a palatalização aplicada a dados /tri, dri/ reduzidos a C1V também estava sob análise, e por essa razão está presente na plotagem.

\section{REFERÊNCIAS}

AVILA, M.C.A.P. A aquisição do ataque silábico complexo: um estudo sobre crianças com idade entre 2:0 e 3:7. Dissertação (Mestrado em Letras) . UCPel, Rio Grande do Sul, 2000.

BAESSO, J.S. O uso de estratégias de reparo nos constituintes coda e ataque complexo por crianças com aquisição fonológica normal e desviante. Dissertação (Mestrado em Distúrbios da Comunicação Humana). Santa Maria, UFSM, 2009.

FIKKERT, P. On the acquisition of prosodic structure. Ph.D. Dissertation (Master in Linguistics), HIL dissertations 6, Leiden University. The Hague: Holland Academic Graphics, 1994. 
FREITAS, M.J. A aquisição da estrutura silábica do português europeu. Lisboa, 1997. 396p. Tese (Doutorado em Linguística) Universidade de Lisboa, Lisboa, 1997. GALEA, D.E.S. Percurso de aquisição dos encontros consonantais, fonemas e estruturas silábicas em crianças de 2;1 a 3;0 anos de idade. Tese (Doutorado em Linguística). Universidade de São Paulo, São Paulo, 2008

GREENLEE, M. Interacting processes in the child's acquisition of stop-liquid clusters. Papers and Reports on Child Language Development. Volume 7, p. 85-100,1974

LAMPRECHT, R.R. A aquisição da fonologia do Português na faixa etária dos 2:9-5:5. Letras de Hoje, v.28, n.2, p.99-106, jun. 1993.

LAMPRECHT, R.R. Antes de mais nada. In: LAMPRECHT, R.R. et al. Aquisição fonológica do português: perfil de desenvolvimento e subsídios para terapia. Porto Alegre: Artmed, 2004. Capítulo 1, p. 17-32.

MATZENAUER, C.L.B \& MIRANDA, A.R.M. A construção do conhecimento fonológico na aquisição da linguagem. Revista de Estudos da Linguagem, Belo Horizonte, v. 20, n. 2, p. 91-124, jul./dez. 2012

RIBAS, L.P. Aquisição do Ataque Complexo. Dissertação (Mestrado em Letras), Porto Alegre: PUCRS, 2002.

SELKIRK, E. The Syllable. In: HULST; SMITH. (eds.). The Structure of Phonological Representations (Part II). Dordrecht Foris, p. 337-383,1982

STAUDT, L. B. Aquisição de onsets complexos por crianças de dois a cinco anos: um estudo longitudinal com base na Teoria da Otimidade. Dissertação (Mestrado em Linguística Aplicada). Ciências da Comunicação, Universidade do Vale do Rio dos Sinos, São Leopoldo, 2008.

TEIXEIRA, E. Processos de simplificação fonológica como parâmetros maturacionais em português. Cadernos de estudos linguísticos, n. 14, p. 53-63, 1988.

TESSIER, A. Stages of Phonological Acquisition and ErrorSelective Learning. In: Baumer, Montero\& Scanlon (orgs), Proceedings of the 25th West Coast Conference on Formal Linguistics. Somerville, MA: Cascadilla Proceedings Project, p. 408-416, 2016.

TONI, A. Representação subjacente do ataque ramificado CCV na aquisição fonológica. Dissertação (Mestrado em Linguística), Universidade de São Paulo, São Paulo, 2016.

Recebido em: 30/04/2017

Aceito em:18/07/2017 